\title{
WALK-OFF AND PATTERN SELECTION IN OPTICAL PARAMETRIC OSCILLATORS
}

\author{
Marco Santagiustina, Pere Colet, Maxi San Miguel, Daniel Walgraef ${ }^{+}$ \\ Instituto Mediterraneo de Estudios Avanzados, IMEDEA (CSIC-UIB)*, \\ E-07071 Palma de Mallorca, Spain
}

\begin{abstract}
The effect of the walk-off in pattern selection in optical parametric oscillators is theoretically examined. We show that a dynamical mechanism allows to observe the formation of structures also for positive signal detunings. In this regime the generated pattern is a periodic array of kinks which separate regions where alternatively one of two stable steady-states is selected. This structure can be regarded as a train of dark stripe solitons because the two solutions have opposite signs. The wavelength of the selected pattern is theoretically predicted and the prediction agrees with the results of the numerical solutions of the equations governing the device.
\end{abstract}

Optical parametric oscillators (OPO's) are one of the nonlinear optical systems where transverse pattern formation has been predicted $^{1}$. Recent experimental results on spatial modulational instability in bulk quadratic media ${ }^{2}$ are very encouraging about the possibility of observing similar pattern formation in a nonlinear resonator. The OPO's have been also investigated because of their properties as generators of squeezed and other non-classical states of the light ${ }^{3}$, which make them a paradigmatic example of the interplay among quantum and macroscopic effects in optical systems ${ }^{4}$. The foreseen applications of these systems are also very valuable, including low-noise measurements and detection ${ }^{3}$ and optical storage 
elements with 2-dimensional localized structures ${ }^{5}$.

Pattern formation was predicted in the OPO's when the pump amplitude is above the signal generation threshold and the detuning between the cavity resonance and the signal frequency is negative ${ }^{1}$. Under this condition the diffraction can compensate the detuning and the homogeneous state becomes unstable for plane-waves which have a small wavevector component in the transversal direction. For positive detunings the steady-state is unstable for homogeneous perturbations and thus no pattern with a characteristic periodicity is predicted. Two homogeneous stable solutions with equal amplitude but opposite phase also exist and then for positive detunings domains can be generated where one of the two solutions is selected. The walls separating the domains are dark soliton stripes as shown in ref. ${ }^{6}$. Here, we study the effect of the walk-off on the pattern formation processes. This phe-

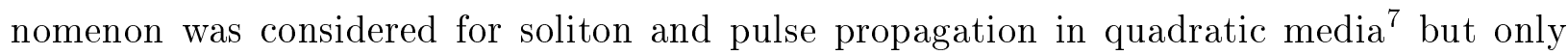
recently its influence has started to be taken into account for OPO's ${ }^{8}$. It stems from the birefringence of the nonlinear crystal which is exploited to phase-match the down-conversion process. Considering a Type I phase-matching scheme, the fundamental and second harmonic waves $(\mathrm{FH}, \mathrm{SH})$ are linearly and orthogonally polarized and therefore, because of the anisotropy, their Pointying vectors are misaligned as soon as the propagation is not parallel to an optical axis, i.e. they walk-off one each other.

Walk-off causes the pattern to drift and forces the orientation of the stripes to be orthogonal to the drift direction; this is due to the fact that the mode orthogonal to the drift motion is the most rapidly spreading in the system ${ }^{8}$. The walk-off also increases the threshold of the absolute instability and a parameter region exists where the instability is convective. In the latter regime the drift motion overwhelms the instability growth and a pattern can be observed only if noise is present ${ }^{8,9}$. Here, we demonstrate that the presence of the walk-off dynamically induces the growth of a spatial pattern also for positive signal detunings. In this case the structure is a periodic array of kinks and the walls separating different domains are dark soliton stripes. We also predict the wavelength of the selected pattern and a very good agreement is found with the results of the numerical solutions of the equations modeling the 
device.

The equations describing the time evolution of the transverse $\mathrm{SH}\left(A_{0}(x, y, t)\right)$ and $\mathrm{FH}$ $\left(A_{1}(x, y, t)\right)$ electric fields in a Type I, phase-matched, degenerate OPO in the mean-field approximation $\operatorname{are}^{1,8}$ :

$\partial_{t} A_{0}=\gamma_{0}\left[-\left(1+i \Delta_{0}\right) A_{0}+E_{0}+i a_{0} \nabla^{2} A_{0}+2 i K_{0} A_{1}^{2}\right]$

$\partial_{t} A_{1}=\gamma_{1}\left[-\left(1+i \Delta_{1}\right) A_{1}+\rho_{1} \partial_{y} A_{1}+i a_{1} \nabla^{2} A_{1}+i K_{0} A_{1}^{*} A_{0}\right]$

where the coefficients $\gamma_{0,1}, \Delta_{0,1}, a_{0,1}, K_{0}$, represent respectively the cavity decay rates, the detunings, the diffraction and the nonlinearity; other parameters are the injected pump $E_{0}$ and the walk-off coefficient $\rho_{1}$.

When $\rho_{1}=0$, a linear stability analysis of the solution $A_{0}=E_{0} /\left(1+i \Delta_{0}\right), A_{1}=0$ shows that for negative signal detunings $\Delta_{1}$ the most unstable modes for $A_{1}$ are travelling waves $\exp \left[i\left(q_{x} x+q_{y} y\right)+\lambda(\vec{q}) t\right]$ with wavevector modulus $|\vec{q}|=q_{c}=\sqrt{-\Delta_{1} / a_{1}}$. They all become unstable when $E_{0}$ is such that $\Re[\lambda]=0$ but only two conjugate waves of this ring of unstable modes will be eventually selected and a stripe structure will arise in the FH intensity field ${ }^{1}$. The final orientation of the standing wave is random, depending only on the initial conditions. For positive detunings the steady-state is unstable for homogeneous perturbations $(\vec{q}=0)$ and the system can evolve to any of the two stable non-zero homogeneous solutions of eqs. $(1,2)^{5,6}$ :

$$
A_{1}^{ \pm}= \pm\left(\frac{I}{2}\right)^{1 / 2}\left(\left(1+\frac{\Delta_{0}+\Delta_{1}}{K_{0} E_{0}}\right)^{1 / 2}+i\left(1-\frac{\Delta_{0}+\Delta_{1}}{K_{0} E_{0}}\right)^{1 / 2}\right)
$$

where the intensity $I=\left|A_{1}^{ \pm}\right|^{2}$ is given by:

$$
I=\frac{1}{2 K_{0}^{2}}\left(\Delta_{0} \Delta_{1}-1+\sqrt{K_{0}^{2} E_{0}^{2}-\left(\Delta_{0}+\Delta_{1}\right)^{2}}\right)
$$

From random initial conditions one observes the growth of spatial domains where the field is either $A_{1}^{+}$or $A_{1}^{-}$. The walls separating these domains are stripes of phase defects, because the amplitude of the field crosses zero. These structures can be also considered as dark solitary waves; their existence and properties have been reported and discussed in ref. ${ }^{6}$. 
As we show here, this scenario drastically changes in presence of the walk-off which induces the creation of a pattern as a dynamical effect. This behaviour is analogous to that of 1-dimensional physical systems in which front propagation into an unstable state creates a pattern behind the front ${ }^{10}$. In the OPO's the injected pump is a beam of finite size and the FH drifts at a given speed $\rho_{1}$. Then, the steady-state $A_{1}=0$ becomes unstable by crossing the boundary where the pump $E_{0}$ reaches the signal generation threshold. The calculation to determine the selected wavevector of the structure is formally the same of refs. ${ }^{10}$. It is based on a linear analysis in which the wavevector is extended to the complex space. In particular, we first determine the wavevector of the most unstable modes by calculating the complex saddle point $\vec{k}_{s}=\left(k_{x}^{s}, k_{y}^{s}\right)$

$\left.\nabla_{\vec{k}} \lambda(\vec{k})\right|_{\vec{k}_{s}}=0, \Re\left[\left.\nabla_{\vec{k}}^{2} \lambda(\vec{k})\right|_{\vec{k}_{s}}\right] \geq 0$

where $\lambda(\vec{k})$, the eigenvalue which results from the linearization of eqs. $(1,2)$ around the steady-state, below threshold, can be written as:

$\lambda(\vec{k})=-1+\rho_{1} k_{y}+\sqrt{F^{2}-a_{1}^{2}\left(q_{c}^{2}+k_{x}^{2}+k_{y}^{2}\right)^{2}}$

and $F=K_{0} E_{0} /\left(1+i \Delta_{0}\right)$. Note that we have made the formal substitution $i \vec{q} \rightarrow \vec{k}$ and thus the wavevector of the plane wave is represented in this notation by $\Im[\vec{k}]$. Equations $(5,6)$ yield $\Im\left[k_{x}^{s}\right]=0$ so that the pattern is expected to be always oriented orthogonally to the drift direction. The $y$ component of the wavevector of the generated pattern, say $Q_{y}$, can be calculated as follows. The linearly unstable wavevector is $\Im\left[k_{y}^{s}\right]$ which oscillates with frequency $\Im\left[\lambda\left(\vec{k}_{s}\right)\right]$ and drifts at velocity $\rho_{1}$. By imposing the conservation of the flux of the field nodes from the linearly unstable regime to the stable state we have ${ }^{10,11}$ :

$Q_{y}=\frac{\Im\left[\lambda\left(\vec{k}_{s}\right)\right]}{\rho_{1}}$

In fig. 1 we present the predicted $Q_{y}$ as a function of the signal detuning calculated for the value of $E_{0}$ at the absolute instability threshold, which is determined by the additional condition $\Re\left[\lambda\left(\vec{k}_{s}\right)\right]=0^{8}$; for comparison, we also include $q_{c}$. Note that for large negative 
detunings the selected wavevector depends on the walk-off only slightly. For small negative detunings the difference increases and the pattern for $\Delta_{1}=0$ has a non-zero wavelength as soon as $\rho_{1} \neq 0$. The predicted $Q_{y}$ decays to zero only for $\Delta_{1} \geq \Delta_{c}\left(\rho_{1}\right)>0$; thus for positive detunings a pattern can form too. In this case the field alternatively assumes the values $A_{1}^{ \pm}$ and a novel kind of optical pattern, i.e. a periodic array of kinks, is predicted.

To confirm this, we have solved numerically eqs. $(1,2)$ with initial random conditions and a flat top pump beam $E_{0}$. Initially we observe the random formation of domains separated by dark stripes and rings ${ }^{6}$. Later, these structures drift outside the pumping region and are replaced by an ordered array of stripes such for example that of fig. 2. The wavevector selected in the numerical solutions is displayed in fig. 1. The $x$ component is zero (see fig. 2 ) and the $y$ component is in a very good agreement with the theory for any $\Delta_{1}$. In fig. 3 we show a transverse section at $x=0$ which exhibits the ordered periodic array of dark solitons. As the detuning increses the regions of constant amplitude get larger and larger and finally, above $\Delta_{c}$, the pattern becomes homogeneous.

In conclusion, we have demonstrated that the walk-off effect in OPO's induces a dynamical selection of the pattern likewise the problem of front propagation into an unstable state. In the OPO the front of the instability is spatially fixed by the injected pump beam and the generated fundamental harmonic field drifts, because of the walk-off. The strength of the walk-off sets the wavelength of the generated pattern and the critical value of the signal detuning $\Delta_{c}$ beyond which no pattern forms. Through this mechanism an array of kinks can be dynamically generated for positive detunings. The characteristic wavevector observed in the numerical solutions is in agreement with the theory for the whole range of parameters. The experimental observation of this phenomenon should be possible with the available technology $y^{6,8}$.

Finally, note that at a fixed position in space, the OPO is generating a temporal train of dark solitons. Then, possible applications of this dynamical pattern formation can be forseen in the field of optical communications where dark soliton trains have been proposed as supporting structures to reduce soliton jitter in optical fiber links ${ }^{12}$. 
This work is supported by QSTRUCT (Project ERB FMRX-CT96-0077). Financial support from DGICYT (Spain) Project PB94-1167 is also acknowledged. 


\section{REFERENCES}

+. Permanent address: Center for Nonlinear Phenomena and Complex Systems, Université Libre de Bruxelles, Campus Plaine, Blv. du Triomphe B.P 231, 1050 Bruxelles.

*. $\quad$ http://www.imedea.uib.es/PhysDept

1. K. Staliunas, Opt. Comm. 91, 82 (1992); J. Mod. Opt. 42, 1261 (1995); G-L. Oppo, M. Brambilla, L.A. Lugiato, Phys. Rev. A, 49, 2028 (1994); G-L. Oppo, M. Brambilla, D. Camesasca, A. Gatti, L.A. Lugiato, J. Mod. Opt. 41, 1151 (1994); S. Longhi, J. Mod. Opt. 43, 1089 (1996); J. Mod. Opt. 43, 1569 (1996); Phys. Rev. A, 53, 4488 (1996); S. Longhi, A. Geraci, Phys. Rev. A, 54, 4581 (1996); G.J. de Varcarcel, K. Staliunas, E. Roldan, V.J. Sanchez-Morcillo, Phys. Rev. A, 54, 1609 (1996); Phys. Rev. A, 56, 3237 (1997).

2. R.A. Fuerst, D-M Baboiu, B.Lawrence, W.E Torruelas, G.I. Stegeman, S. Trillo, Phys. Rev. Lett. 78, 2756 (1997);

3. H.J. Kimble, Fundamental systems in quantum optics (J. Dalibard, J.M. Raimond, J. Zinn-Justine, Elsevier Sc., Amsterdam) 545 (1992).

4. L.A. Lugiato, A. Gatti, H. Wiedemann, Quantum Fluctuations (S. Reynaud, E. Giacobino, J. Zinn-Justine Eds., Elsevier Sc., Amsterdam), 431 (1997).

5. K. Staliunas, V.J. Sanchez-Morcillo, Opt. Comm., 139, 306 (1997), Phys. Rev. A 57, 1454; M. Tlidi, P. Mandel, M. Haelterman, Phys. Rev. E, 56, 6524 (1998).

6. S. Trillo, M. Haelterman, A. Sheppard, Opt. Lett. 22, 970 (1997).

7. W.E. Torruelas, Z. Wang, D.J. Hagan, E.W. Van Stryland, G.I. Stegeman, L. Torner, C.R. Menyuk, Phys. Rev. Lett., 74, 5036 (1995); A.V. Smith, W.J. Alford, T.R. Raymond, M.S. Bowers, J. Opt. Soc. Am. B 12, 2253 (1995).

8. M. Santagiustina, P. Colet, M. San Miguel, D. Walgraef, submitted (1998). 
9. M. Santagiustina, P. Colet, M. San Miguel, D. Walgraef, Phys. Rev. Lett., 79, 3363 (1997).

10. G.T. Dee, W. Van Sarloos, Phys. Rev. Letts., 60, 2641 (1988).

11. R. Montagne, A. Amengual, E. Hernandez-Garcia, M. San Miguel, Phys. Rev. E, 50, 377 (1994); M. San Miguel, R. Montagne, A. Amengual, E. Hernandez-Garcia, Instabilities and Nonequilibrium Structures V, 85 (E. Tirapegui, W. Zeller Eds., Kluwer Ac., Amsterdam) (1996).

12. P.D. Miller, N.N. Akhmediev, A. Ankiewicz, Opt.Lett. 21, 1132 (1996). 
Figure Captions:

Figure 1: The predicted wavevector of the pattern is presented in solid line for $\rho_{1}=0.15$. The asterisks represent the wavevector selected in the numerical solutions and the dashed line the predicted wavevector for $\rho_{1}=0$. Numerical solutions are calculated for $E_{0}$ always $1 \%$ above the absolute instability threshold (see text) and $\Delta_{0}=0$. We also set $\gamma_{0,1}=1$, $a_{0}=a_{1} / 2=0.125, K_{0}=1$.

Figure 3: The transverse intensity pattern at $t=2000$ is shown; the parameters used are $\Delta_{0}=0, \Delta_{1}=0.3, \rho_{1}=0.15, E_{0}=1.12736, \gamma_{0,1}=1, a_{0}=a_{1} / 2=0.125, K_{0}=1$.

Figure 3: The real (solid line) and imaginary (dashed line) parts of the electric field envelope in function of the space variable $y$ are given for $x=0$. The value of the uniform regions agree with the solutions predicted by eq. (4), $A_{1}^{ \pm}= \pm(0.166+i 0.126)$. Other parameters are as in fig. 2 . 


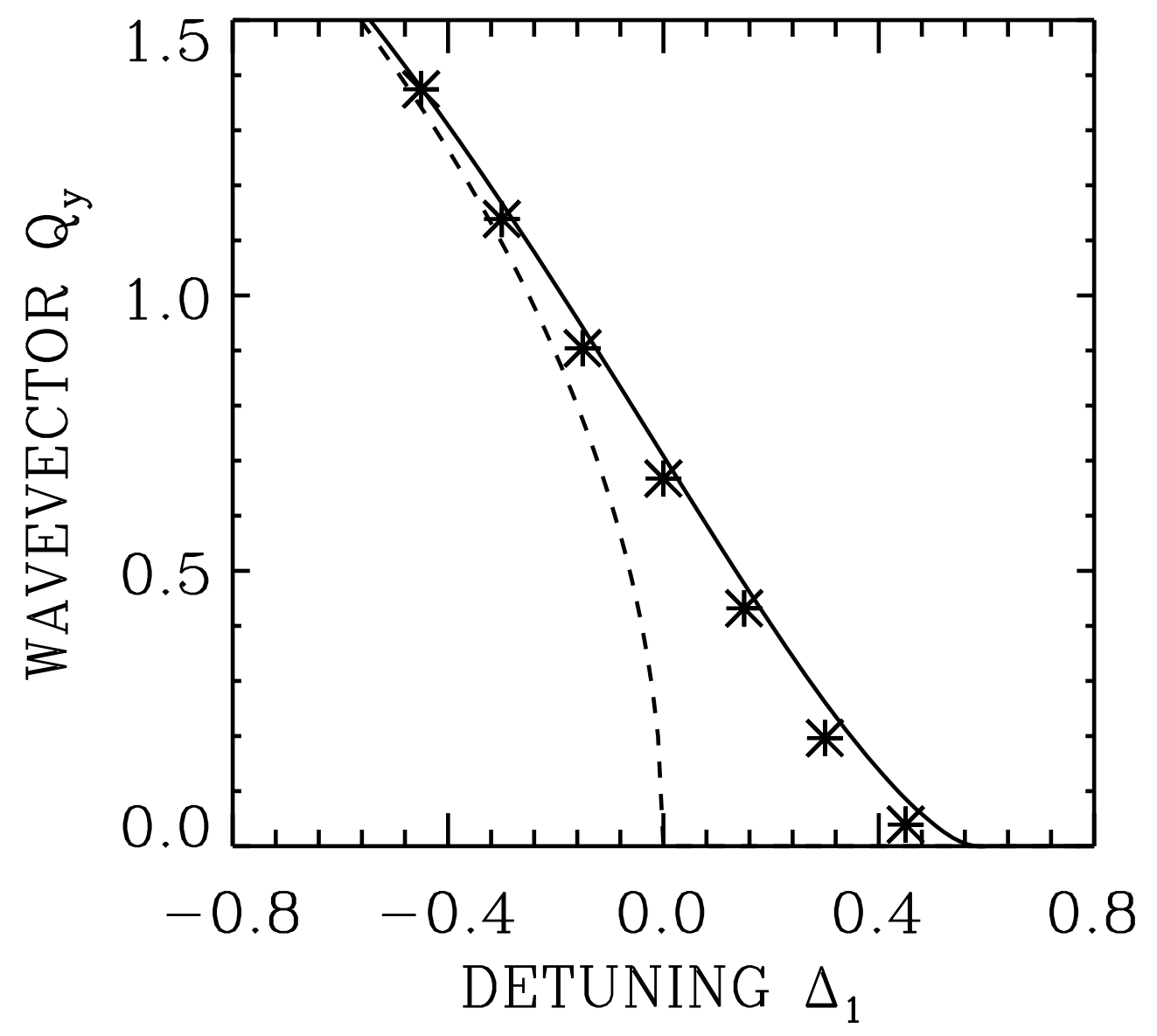




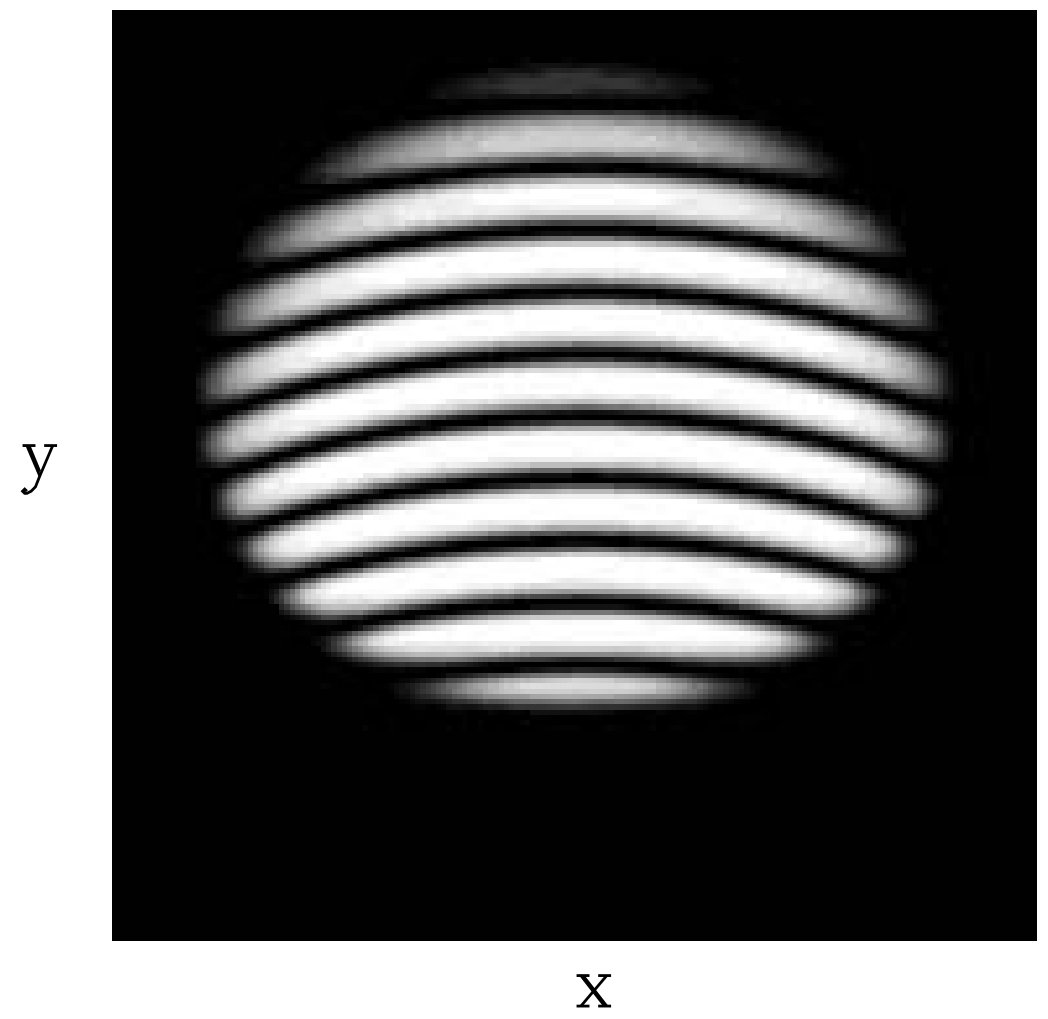




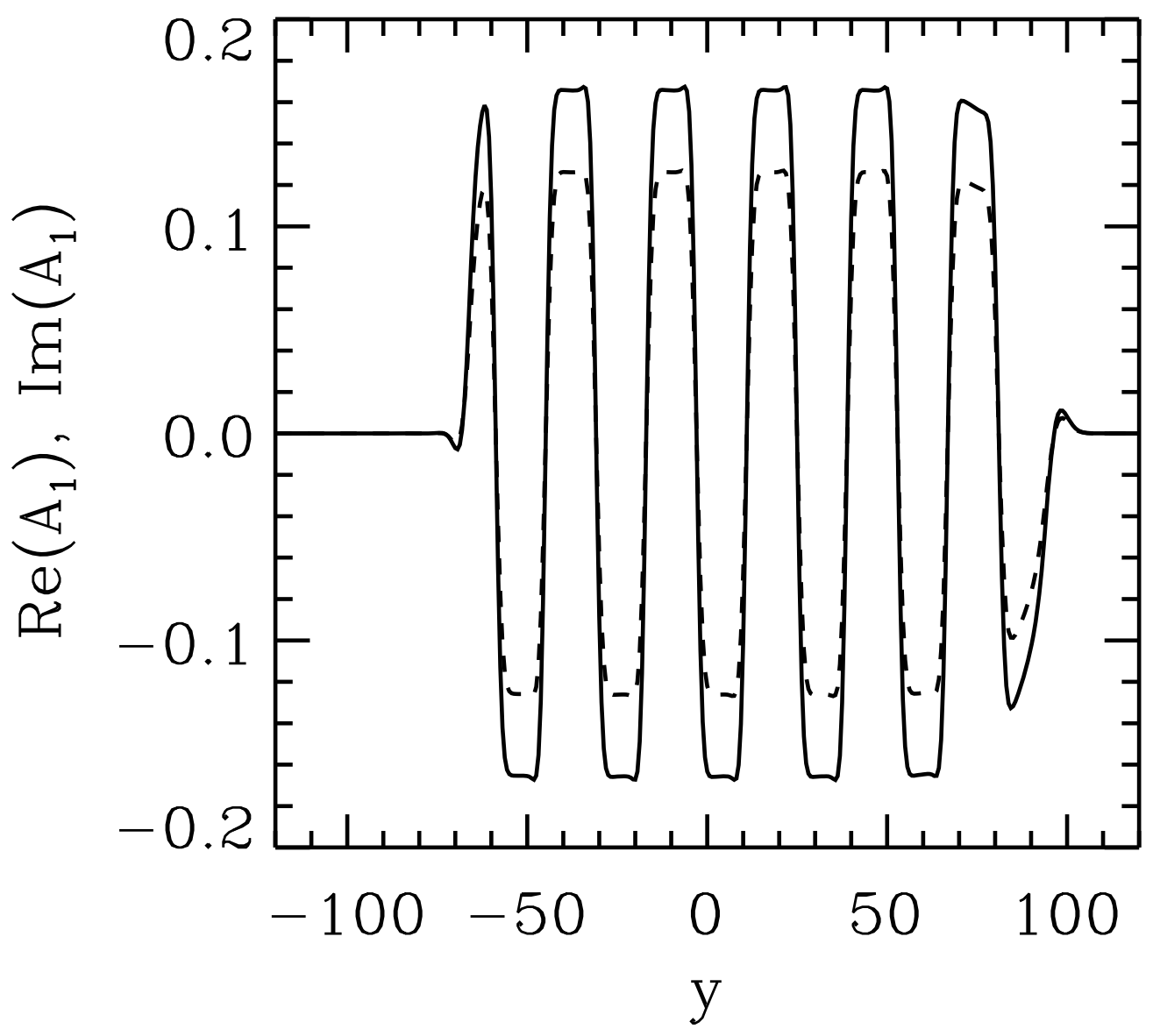

